\title{
Optimal Cycles and Social Inequality: What Do We Learn from the Gini Index?*
}

\author{
Stefano BOSI ${ }^{\dagger}$ and Thomas SEEGMULLER ${ }^{\ddagger}$
}

January 4, 2006

\begin{abstract}
One of the plausible explanations for macroeconomic fluctuations relies on the occurrence of endogenous deterministic cycles. In the last three decades, most of the relevant literature has rested on the assumption of a representative agent but, recently, a few papers have investigated the role of consumers' heterogeneity on endogenous fluctuations. Our article aims at taking a step forward in order to give a more suitable interpretation. To keep things as simple as possible, we introduce heterogeneous households in a two-sector optimal growth model and we study how wealth heterogeneity affects the occurrence of endogenous cycles. In contrast to previous results, we relate the existence of such cycles to the most commonly used inequality measure, the Gini index, and analyze the impact of consumers' heterogeneity on this index.
\end{abstract}

JEL classification: D31, E32, O41.

Keywords: Endogenous cycles, two-sector models, heterogeneous agents, Gini index.

\section{Introduction}

One of the puzzling questions the economists are addressed is whether income inequality can be pointed out as a catalyzer of macroeconomic instability, or, more explicitly, whether less developed countries with higher income inequality are less sheltered from economic fluctuations and suffer a wider macroeconomic volatility. In this paper, we aim at providing a theoretical answer, by considering

\footnotetext{
*We would like to thank all the participants to the conference "Intertemporal Equilibria, Aggregation and Sunspots: in Honor of Jean-Michel Grandmont" held in Lisbon on October 2005 and, in particular, Carine Nourry and Alain Venditti.

†EPEE, Université d'Evry, 4, bd F. Mitterrand, 91025 Evry cedex, France. Tel: + 33169 4770 52. Fax: + 331694770 50. E-mail: stefano.bosi@univ-evry.fr.

$\ddagger$ CNRS and EUREQua, 106-112, bd. de l'Hôpital, 75647 Paris cedex 13, France. Tel: + 331440781 99. Fax: + 331440782 31. E-mail: seegmu@univ-paris1.fr.
} 
a usual neoclassical dynamic framework, where volatility is interpreted in terms of endogenous cycles.

Well-known features of the one-sector optimal growth models are the uniqueness of the steady state and the saddle-path stability. The monotonic convergence to the steady state is no longer ensured in multi-sector models. Benhabib and Nishimura (1985) have shown that oscillations and two-period cycles can arise in a two-sector framework. In their model, the consumption and the investment good are produced through different technologies. Such a productive heterogeneity plays an essential role in the emergence of cycles, since the consumption good is required to be more capital intensive than the investment good. Nevertheless, most of this literature, which is concerned with the existence of competitive cycles and endogenous fluctuations, fails to introduce heterogeneity on the consumption side of the economy and takes the shortcut of a representative agent.

Recently, Herrendorf et al. (2000) have addressed some criticism to the occurrence of expectations-driven fluctuations under the assumption of a representative agent. Surprisingly, they prove that a degree of agents' heterogeneity can rule out indeterminacy. However, they deal with a quite peculiar model, where nominal rigidities matter. Thus, the robustness of their findings has been the subject of further studies since. A few authors have introduced heterogenous households in dynamic general equilibrium models.

Ghiglino and Olszak-Duquenne (2001), Ghiglino (2005) and Ghiglino and Venditti (2005) have focused on the influence of heterogeneity on the occurrence of optimal cycles and non-monotonic trajectories. In these papers, households are heterogeneous, because they are represented by different utility functions and are unequally endowed with capital and labor. In order to show the effects of heterogeneity on the equilibrium dynamic properties, they exploit the market perfection and characterize directly the Pareto-optimal allocations without caring about the decentralized solution, by using the existence and continuity of the welfare weights with respect to initial conditions (see Kehoe et al. (1990) and Santos (1992)).

Ghiglino (2005) and Ghiglino and Venditti (2005) find that the influence of inequality and heterogeneity on macroeconomic (in)stability depends on the concavity (convexity) of the absolute risk tolerance, that is the inverse of the absolute risk aversion. Despite the findings are rich and precise, they remain difficult to understand and to interpret in terms of heterogeneity at an individual level: these authors don't consider popular aggregate inequality measures. In addition, several original results obtained in these papers no longer apply to more consensual utility functions characterized by constant elasticities of intertemporal substitution in consumption.

Our paper takes into account these criticisms, by targeting and reexamining the connection between consumers' heterogeneity and stability properties in an optimal growth two-sector model, where consumers' preferences are characterized by a constant elasticity of intertemporal substitution. We will discuss not only the role of wealth inequality on dynamics, but also that of preferences heterogeneity. We connect the emergence of optimal cycles to the well-known in- 
equality measure, the Gini index, and analyze how it is affected by heterogeneity in preferences.

We focus directly on the decentralized equilibrium, instead of considering the Pareto allocations. The stationary solution is shown to depend on the sharing of initial endowments and, therefore, is formally characterized by the presence of a unit root. Such a feature is not a curiosity, but rather a general property of an heterogeneous world.

In line with Benhabib and Nishimura (1985), we prove that endogenous cycles cannot arise when the investment good is relatively more capital intensive, while non-monotonic trajectories can occur if the pure consumption good is more capital intensive. In this case we are able to determine the critical distribution of capital stock beyond which two-period cycles and period-doubling flip bifurcations occur. Moreover, in contrast to the existing papers, we introduce a commonly used measure of inequality, the Gini index, the equilibrium value of which mainly depends on the wealth sharing. We find the critical Gini degree corresponding to the critical distribution of capital and we explain how it varies in response to changes in preferences heterogeneity, more precisely when heterogeneity in consumers' preferences raises. More heterogeneity in consumers' preferences raises or lowers the Gini index, according to the interplay between the substitution and income effects, the relative importance of which is determined by the consumers' average elasticity of intertemporal substitution.

In sum, optimal cycles occur under well-known technological conditions in our two-sector economy. However, in contrast to the existing literature, we provide clear-cut insights about the role of consumers' heterogeneity on the emergence of persistent cycles. Indeed, heterogeneity in preferences interferes with the technological source of cycles, through the unequal capital distribution. Eventually, we connect the appearance of cycles to the Gini index, one of the most commonly used measures of inequality. Hence, the role of heterogeneity on optimal cycles is revisited from a point of view perhaps much simpler than the existing approaches, but probably more understandable.

The rest of the paper is organized as follows. Section 2 and 3 are devoted to present, respectively, the behavior of heterogeneous consumers and producers. In section 4 , we determine the equilibrium and analyze the steady state. In section 5, we define the measure of inequality we will use later: the Gini index. Section 6 presents the stability properties of the steady state and the conditions under which cycles occur. We also relate the flip bifurcation to the Gini index. In section 7 , the impact of heterogeneous preferences on the critical Gini index is discussed. Concluding remarks are provided in the last section, whereas technicalities are gathered in the Appendix.

\section{Heterogeneous consumers}

We consider an economy with a constant population $l$ of infinite-lived agents over a discrete time $t=0,1, \ldots, \infty$. There are two types of consumers $(i=1,2)$, who differ by their capital endowments and their preferences. More precisely, 
there is a continuum $\left[0, l_{1}\right]$ of agents of type 1 (say, the poor), each one endowed with an amount $\tilde{k}_{1}$ of capital, and a continuum $\left(l_{1}, l\right]$ of agents of type 2 (the rich), endowed with an amount $\tilde{k}_{2}>\tilde{k}_{1}$. Whatever the class he belongs, each household supplies inelastically one unit of labor at each period.

Two goods are produced and exchanged in this economy: a consumption good, which plays also the role of numéraire, and an investment good, which is sold at the price $p_{t}$. The real interest rate $r_{t}$ and the real wage $w_{t}$ are measured in consumption good units, while $\Delta \equiv 1-\delta \in(0,1)$, where $\delta \in(0,1)$ is the rate of capital depreciation. At each period, a consumer of type $i$ faces a budget constraint:

$$
\tilde{c}_{i t}+p_{t}\left(\tilde{k}_{i t+1}-\Delta \tilde{k}_{i t}\right) \leq r_{t} \tilde{k}_{i t}+w_{t}
$$

where $\tilde{c}_{i t}$ represents his consumption at time $t$. His intertemporal preferences are summarized by an utility function:

$$
\sum_{t=0}^{\infty} \beta^{t} \frac{\tilde{c}_{i t}^{1-1 / \sigma_{i}}}{1-1 / \sigma_{i}}
$$

where $\beta \in(0,1)$ is a common discount factor to both agents' types. $\sigma_{i}>0$ $\left(\sigma_{i} \neq 1\right)$ is the elasticity of intertemporal substitution in consumption and captures heterogeneity in preferences as soon as $\sigma_{1} \neq \sigma_{2}$.

The consumer maximizes his utility function (2), taking the budget constraint (1) as given. We derive the Euler equation:

$$
\frac{\tilde{c}_{i t+1}}{\tilde{c}_{i t}}=\left(\beta \frac{r_{t+1}+\Delta p_{t+1}}{p_{t}}\right)^{\sigma_{i}}
$$

and the budget constraint (1), now binding. The transversality condition is also required to be satisfied by the sequence of individual demands: $\lim _{t \rightarrow \infty} \lambda_{t} \tilde{k}_{i t+1}=$ 0 .

Aggregating equations (3) across the individuals of the same type and setting $c_{i} \equiv \tilde{c}_{i} l_{i}, k_{i} \equiv \tilde{k}_{i} l_{i}$ with $i=1,2$, we find two equations: ${ }^{1}$

$$
\begin{aligned}
\frac{c_{i t+1}}{c_{i t}} & =\left(\beta \frac{r_{t+1}+\Delta p_{t+1}}{p_{t}}\right)^{\sigma_{i}} \\
r_{t} k_{i t}+w_{t} l_{i} & =c_{i t}+p_{t}\left(k_{i t+1}-\Delta k_{i t}\right)
\end{aligned}
$$

\section{Heterogeneous producers}

The amounts of the pure consumption good and the capital good are given, respectively, by $c$ and $y$ and are produced with two different technologies: $c=$ $F^{1}\left(k^{1}, l^{1}\right)$ and $y=F^{2}\left(k^{2}, l^{2}\right)$, where $k^{j}$ and $l^{j}$ denote the inputs processed in each sector. The aggregate factors demands are given by $k=k^{1}+k^{2}$ and

\footnotetext{
${ }^{1}$ Notice that $l_{2}$ is defined by $l_{2} \equiv l-l_{1}$.
} 
$l=l^{1}+l^{2}$. The derivatives of $F^{j}$ with respect to $k^{j}$ and $l^{j}$ are respectively denoted $F_{1}^{j}$ and $F_{2}^{j}$. We further assume:

Assumption 1 Each production function $F^{j}: R_{+}^{2} \rightarrow R_{+}, j=1,2$, is $C^{2}$, increasing in each argument, concave, homogeneous of degree one and such that, for any $x>0, F^{j}(0, x)=F^{j}(x, 0)=0, F_{1}^{j}(0, x)=F_{2}^{j}(x, 0)=+\infty$, $F_{1}^{j}(+\infty, x)=F_{2}^{j}(x,+\infty)=0$.

Under this assumption, we can apply the implicit function theorem to $y=$ $F^{2}\left(k^{2}, l^{2}\right)$, in order to obtain a locally explicit function $k^{2}\left(y, l^{2}\right)$. Replacing $k^{1}=k-k^{2}\left(y, l^{2}\right)$ and $l^{1}=l-l^{2}$ in the production function of the consumption sector and maximizing the output $c$ with respect to $l^{2}$, given $k, y$ and $l$, we get eventually the value $c=\max _{l^{2}} F^{1}\left(k-k^{2}\left(y, l^{2}\right), l-l^{2}\right) \equiv T(k, y, l)$, which defines a technological frontier. Applying the envelope theorem to the value, we compute the slope of the transformation frontier:

$$
\begin{aligned}
& T_{1}(k, y, l)=r \equiv r(k, y, l) \\
& T_{2}(k, y, l)=-p \equiv-p(k, y, l) \\
& T_{3}(k, y, l)=w \equiv w(k, y, l)
\end{aligned}
$$

where $T_{i}$ is the partial derivative of the function $T$ with respect to the $i$ th argument. ${ }^{2}$ The concavity of the frontier is also characterized, but the reader is referred, for the sake of brevity, to the seminal Benhabib and Nishimura (1985) or to Bosi, Magris and Venditti (2005) for more computational details:

$$
\begin{aligned}
& T_{11}=\partial r(k, y, l) / \partial k<0 \\
& T_{22}=-\partial p(k, y, l) / \partial y=b^{2} T_{11}<0 \\
& T_{12}=\partial r(k, y, l) / \partial y=T_{21}=-\partial p(k, y, l) / \partial k=-b T_{11}
\end{aligned}
$$

where $b \equiv\left(k^{2} / l^{2}-k^{1} / l^{1}\right) l^{2} / y$ is the relative capital intensity difference across sectors: a simple, but indispensable parameter to understand the technological heterogeneity. We notice that $b$ determines the sign of $T_{12}$ and that the investment good is more capital intensive when $b$ is positive, while, on the opposite, the consumption good is more capital intensive when $b$ is negative.

\section{Equilibrium}

An intertemporal equilibrium requires, at each period, that the inputs markets on the one side and the outputs markets on the other side clear. Equilibrium on the capital market requires $k_{t}^{1}+k_{t}^{2}=k_{1 t}+k_{2 t}=k_{t}$, while on the labor market requires $l_{t}^{1}+l_{t}^{2}=l_{1}+l_{2}=l$. Since $T\left(k_{t}, y_{t}, l\right)$ represents the supply of consumption good and $y_{t}$ the produced amount of investment good, both the markets clear, if and only if:

$$
\begin{aligned}
c_{1 t}+c_{2 t} & =T\left(k_{t}, y_{t}, l\right) \\
y_{t} & =k_{t+1}-\Delta k_{t}
\end{aligned}
$$

\footnotetext{
${ }^{2}$ The derivation of equations (6), (7) and (8) is provided in the Appendix.
} 
The consumers' and the producers' optimal plans (4)-(5) and (6)-(8), respectively, jointly with all these equilibrium conditions, define a competitive equilibrium, the properties of which will be characterized in Section 6 .

\subsection{Dynamic system}

Combining consumers' systems (4)-(5), producers' solutions (6)-(7) and equilibrium conditions (10)-(11), we obtain, eventually, a three-dimensional dynamic system:

$$
\begin{aligned}
y_{t} & =k_{t+1}-\Delta k_{t} \\
\frac{c_{1 t+1}}{c_{1 t}} & =\left(\beta\left[\frac{\Delta T_{2}\left(k_{t+1}, y_{t+1}, l\right)-T_{1}\left(k_{t+1}, y_{t+1}, l\right)}{T_{2}\left(k_{t}, y_{t}, l\right)}\right]\right)^{(12)} \begin{array}{l}
\sigma_{1} \\
(13)
\end{array} \\
\frac{T\left(k_{t+1}, y_{t+1}, l\right)-c_{1 t+1}}{T\left(k_{t}, y_{t}, l\right)-c_{1 t}} & =\left(\beta\left[\frac{\Delta T_{2}\left(k_{t+1}, y_{t+1}, l\right)-T_{1}\left(k_{t+1}, y_{t+1}, l\right)}{T_{2}\left(k_{t}, y_{t}, l\right)}\right]\right)^{\sigma_{2}}(14)
\end{aligned}
$$

with $k_{0}>0$ given. These three equations determine the sequence $\left(k_{t}, y_{t}, c_{1 t}\right) \in$ $R_{++}^{3}$ for all $t$, which governs entirely the dynamics of the economy.

\subsection{Steady state}

A steady state is defined as a solution $k_{t}=k_{t+1}=k, y_{t}=y_{t+1}=y$ and $c_{1 t}=c_{1 t+1}=c_{1}$, that satisfies equations (12)-(14). From equation (12), we deduce $y=\delta k$. Putting this value in equation (13), we obtain:

$$
-\frac{T_{1}(k, \delta k, l)}{T_{2}(k, \delta k, l)}=\rho
$$

where $\rho \equiv 1 / \beta-\Delta$. As in the two-sector model with a representative household, we get a standard result:

Proposition 1 Under Assumption 1, there exists a unique steady state $k>0$ solution of equation (15).

Proof. See Becker and Tsyganov (2002).

A solution $k$ corresponds to the Modified Golden Rule: there are no imperfection and dynamics are optimal. The stationary aggregate consumption is defined by $c_{1}+c_{2}=T(k, \delta k, l)$.

We observe that neither the aggregate capital $k$, nor $r, p, w$ are affected by the initial conditions, while $c_{1}$ is, as well as the equilibrium transition path. Indeed, using (5)-(8), $c_{1}$ can be explicitly written: $c_{1}=\left[\left(T_{1}+\delta T_{2}\right) k_{1}+T_{3} l_{1}\right]$. The stationary sharing $\left(k_{1}, k_{2}\right)$ and, then, $\left(c_{1}, c_{2}\right)$ will depend on the initial condition $\left(k_{10}, k_{20}\right)$.

Before defining the inequality measure, let's introduce a useful notations to simplify formulas. We make use of the share of consumption good consumed by the first agent's type: $c_{1} /\left(c_{1}+c_{2}\right)$ or, equivalently,

$$
\varkappa \equiv c_{1} / T \in(0,1)
$$


Market clearing equations allow us to explicitly compute $\varkappa$. Substituting equations (5), (6), (7) and the equilibrium condition (10) in (16), we get:

$$
\varkappa=\lambda-(\lambda-\kappa)(1-\delta / \rho) \varepsilon_{1}
$$

where

$$
\varepsilon_{1} \equiv k T_{1} / T \in(0,1)
$$

while

$$
\begin{aligned}
\kappa & \equiv k_{1} /\left(k_{1}+k_{2}\right) \\
\lambda & \equiv l_{1} /\left(l_{1}+l_{2}\right)
\end{aligned}
$$

are the poor's shares of capital and labor supply, respectively. We further observe that since $\tilde{k}_{1} \leq \tilde{k}_{2}$ and $k_{i}=\tilde{k}_{i} l_{i}$, we obtain $k_{1} / k_{2} \leq l_{1} / l_{2}$ or, equivalently,

$$
0 \leq \kappa \leq \lambda
$$

which implies that $\varkappa \in[0, \lambda]$.

\section{Inequality measure: the Gini index}

In this section, we apply the most widespread measure of social inequality: the Gini index. The existing papers about the role of heterogeneity on the occurrence of optimal cycles (Ghiglino and Olszak-Duquenne (2001), Ghiglino (2005) and Ghiglino and Venditti (2005)), instead of taking in account this simple measure, provide more complicate indicators, such as the absolute risk tolerance of the social utility function. ${ }^{3}$ Ghiglino (2005) and Ghiglino and Venditti (2005) show that inequality favors macroeconomic volatility, when the absolute risk tolerance is a strictly convex function. In the opposite case, equality favors macroeconomic volatility.

However, in order to measure income inequality in a country and compare this phenomenon over time and among countries, economists usually make use of Lorentz curves and Gini indexes. A Lorentz curve plots the cumulative percentages of total income received against the cumulative percentages of recipients, starting with the poorest agent. The Gini index is calculated as the area between a Lorentz curve and the line of absolute equality, expressed as a percentage of the triangle under the line, and is a pure number, independent on the measure unit. Thus a Gini index of 0 represents perfect equality and the Lorentz curve coincides with the straight line of absolute equality.

To have a rough idea about income inequalities, we provide the Gini index for some countries in accordance with the OECD statistics. In 1989 this measure was about $36 \%$ for the OECD countries. The Slovak Republic and Brazil experienced a Gini rate of, respectively, 20\% in 1992 and 63\% in 1989. France

\footnotetext{
${ }^{3}$ The social absolute risk tolerance is defined as the inverse of the social absolute risk aversion.
} 
was at $33 \%$ in 1989, while United States and China were at, respectively, $40 \%$ in 1994 and $41 \%$ in 1995.

Formal simplicity and economic pertinence of the index justify why we build it up as a reduced key parameter in order to study the dynamic properties of the model and local bifurcations.

In general, after ranking the agents $h$ in $[0, l]$ according to their increasing income and denoting the cumulative income by $R(h)$ we define the Gini index as

$$
g=1-2 \frac{\int_{0}^{l} R(h) d h}{R(l) l}
$$

Let's recall that, in our economy, there are $l_{1}$ (poor) agents endowed with $\widetilde{k}_{1}$ and $l_{2}=l-l_{1}$ (rich) agents endowed with $\widetilde{k}_{2}>\widetilde{k}_{1}$. The aggregate income $R_{i}$ of the $i$ th class is given by:

$$
R_{i} \equiv r k_{i}+w l_{i}
$$

with $R_{2} / l_{2}>R_{1} / l_{1}$. Therefore, in our linear case, $R(h)$ is given by $h R_{1} / l_{1}$, if $0 \leq x \leq l_{1}$, and by $R_{1}+\left(h-l_{1}\right) R_{2} / l_{2}$, if $l_{1}<h \leq l$. Replacing this function in (22), we obtain a simple formula:

$$
g=\lambda-R_{1} /\left(R_{1}+R_{2}\right)
$$

Using notations (18), (19), (20) and (23), the ratio $R_{1} /\left(R_{1}+R_{2}\right)$ can be computed from the definitions of prices (6) and (7) on one hand, and the equilibrium conditions (10) and (11) on the other hand:

$$
\frac{R_{1}}{R_{1}+R_{2}}=\frac{\rho \varkappa+\delta \varepsilon_{1} \kappa}{\rho+\delta \varepsilon_{1}}
$$

where $\varkappa$ is given by (17). Combining (24) and (25), we find the equilibrium expression of the Gini index:

$$
g=\frac{\rho \varepsilon_{1}}{\rho+\delta \varepsilon_{1}}(\lambda-\kappa)=\frac{\rho}{\rho-\delta} \frac{\rho}{\rho+\delta \varepsilon_{1}}(\lambda-\varkappa)
$$

\section{Saddle-path stability and optimal cycles}

In this section, we first analyze the stability properties of the steady state and establish the conditions for the occurrence of a flip bifurcation, corresponding to a critical value of capital distribution between the consumers' types. Afterwards, we will connect this result with the Gini index.

We linearize the dynamic system (12)-(14) in a neighborhood of the steady state. We obtain the linear form

$$
\left(d k_{t+1} / k, d y_{t+1} / y, d c_{1 t+1} / c_{1}\right)^{T}=J\left(d k_{t} / k, d y_{t} / y, d c_{1 t} / c_{1}\right)^{T}
$$


where the Jacobian matrix is explicitly given by:

$$
\begin{aligned}
J= & {\left[\begin{array}{ccc}
-\beta(1+\Delta b) \sigma_{1} & \delta \beta b(1+\Delta b) \sigma_{1} & \left(\rho \varepsilon_{2}\right)^{-1} \\
\beta(1+\Delta b) A-\varepsilon_{1} & \delta\left[\varepsilon_{1} / \rho-\beta b(1+\Delta b) A\right] & \varkappa \\
1 & 0 & 0
\end{array}\right] } \\
& {\left[\begin{array}{ccc}
-b \sigma_{1} & \delta b^{2} \sigma_{1} & \left(\rho \varepsilon_{2}\right)^{-1} \\
b A-\varepsilon_{1} & \delta\left(\varepsilon_{1} / \rho-b^{2} A\right) & \varkappa \\
\Delta & \delta & 0
\end{array}\right] }
\end{aligned}
$$

with $A \equiv(1-\varkappa) \sigma_{2} \rho \varepsilon_{2}$ and $\varepsilon_{2} \equiv k T_{11} / T_{1}<0$, the elasticity of the interest rate with respect to the capital.

The characteristic polynomial gets the form $P(\alpha)=\alpha^{3}-T \alpha^{2}+M \alpha-D$, where $T, M$ and $D$ denote, respectively, the trace, the sum of the second order principal minors and the determinant. In order to locate the characteristic roots, we evaluate the polynomial at $\alpha=-1,0,1$.

$$
\begin{aligned}
P(1) & =0 \\
P(0) & =-\operatorname{det} J=-1 / \beta \\
P(-1) & =-2 \frac{\sigma(1+(1+\Delta) b)[1+(1 / \beta+\Delta) b]-2(1+\beta) E}{\sigma b(1+\Delta b)-\beta E}
\end{aligned}
$$

where

$$
\sigma \equiv \varkappa \sigma_{1}+(1-\varkappa) \sigma_{2}
$$

is the average elasticity of intertemporal substitution and

$$
E \equiv\left(\varepsilon_{1} / \varepsilon_{2}\right) /(\beta \rho)^{2}<0
$$

is a technological feature.

The polynomial feature deserves some comments. First, we observe that $D=1 / \beta$ as in the Cass-Koopmans benchmark and in Benhabib and Nishimura (1985): consumers' heterogeneity and social inequality are not imperfections and the equilibrium remains optimal even when trajectories display cyclical properties. Second, $P(1)=0$ entails that $\alpha=1$ is the unit root we were finding, deeply related to the existence of a continuum of steady states (stationary consumption sharing), which are determined, as seen above, by the initial capital sharing.

When there are no market imperfections and the investment sector is more intensive in capital, there is no space for optimal cycles. Cycles appear only when the consumption sector becomes more intensive in capital. The robustness of such a conjecture is now confirmed.

In order to present the main proposition, we set the critical values for the average elasticity of intertemporal substitution:

$$
\begin{aligned}
\sigma^{*} & \equiv \frac{2(1+\beta) E}{[1+(1+\Delta) b][1+(1 / \beta+\Delta) b]} \\
\sigma^{* *} & \equiv \frac{\beta E}{b(1+\Delta b)}
\end{aligned}
$$


We observe that, if $-1 /(1+\Delta)<b<-1 /(1 / \beta+\Delta)$, then $0<\sigma^{* *}<\sigma^{*}$. We are now able to characterize the stability properties of the steady state and the occurrence of endogenous fluctuations:

Proposition 2 In order to ensure prices positivity, we assume $b<1 / \rho$. Then, the stability properties of the steady state are the following.

(1) If $-\infty<b<-1 / \Delta$, the steady state is a saddle and there is monotonic convergence.

(2) If $-1 / \Delta<b<-1 /(1+\Delta)$, the steady state is a saddle. There is monotonic convergence for $\sigma<\sigma^{* *}$ and oscillating convergence for $\sigma^{* *}<\sigma$.

(3) If $-1 /(1+\Delta)<b<-1 /(1 / \beta+\Delta)$, the steady state is a saddle and there is monotonic convergence for $\sigma<\sigma^{* *}$, the steady state is a saddle and there is oscillating convergence for $\sigma^{* *}<\sigma<\sigma^{*}$ and the steady state is a source for $\sigma^{*}<\sigma$. When $\sigma$ goes through $\sigma^{*}$, the system undergoes a flip bifurcation.

(4) If $-1 /(1 / \beta+\Delta)<b<0$, the steady state is a saddle. There is monotonic convergence for $\sigma<\sigma^{* *}$ and oscillating convergence for $\sigma^{* *}<\sigma$.

(5) If $0 \leq b<1 / \rho$, the steady state is a saddle and there is monotonic convergence.

Proof. See the Appendix.

We observe that Proposition (2) focuses on the saddle-path stability (monotonic or oscillating), instability and occurrence of persistent cycles. We provide in the Appendix a detailed presentation of the relative position of the eigenvalues with respect to the unit circle in all these cases.

We notice that when the capital good is more capital-intensive $(b>0)$ or we deal with the one-sector specification $(b=0)$, case 5 holds and convergence turns out to be monotonic: there is no room for endogenous cycles. On the contrary, when $b<0$, endogenous fluctuations can occur. Indeed, optimal cycles arise through a flip bifurcation when $b \in(-1 /(1+\Delta),-1 /(1 / \beta+\Delta))$. As in the two-sector model with a representative agent, the existence of endogenous fluctuations thus requires a more capital intensive consumption good.

The intuition for this result, initially provided by Benhabib and Nishimura (1985) in a two-sector model with homogeneous agents, comes from the Rybcszynski and Stolper-Samuelson effects (see also Drugeon (1999) or Bosi, Magris and Venditti (2005)). ${ }^{4}$ The occurrence of deterministic fluctuations follows a straightforward rationale. For simplicity, let's consider an instantaneous increase in the capital stock $k_{t}$. This results in two opposing forces. (i) Since the consumption good is more capital intensive than the investment good, the trade-off in production becomes more favorable to the consumption good. The Rybcszynski theorem implies also a decrease of the output of the capital good $y_{t}$.

\footnotetext{
${ }^{4}$ The Rybcszynski effect focuses on quantities to explain the emergence of cycles: if the endowment of a factor increases, then the production of the good that uses this factor relatively more intensively raises and the production of the other good decreases. The Stolper-Samuelson effect is a dual theory referring to price mechanics: when the price of the good $j$ increases, the equilibrium price of the factor more intensively used in the production of good $j$ increases too, while the price of the other factor decreases.
} 
This tends to lower the investment and the capital stock in the next period $k_{t+1}$. (ii) In the next period the decrease of $k_{t+1}$ implies again through the Rybcszynski effect an increase of the output of the capital good $y_{t+1}$. This mechanism is explained by the fact that the decrease of $k_{t+1}$ improves the trade-off in production in favor of the investment good which is relatively less intensive in capital. Therefore this tends to increase the investment and the capital stock in period $t+2, k_{t+2}$. Notice also that the rise of $y_{t+1}$ implies a decrease of the rental rate $r_{t+1}$ and an increase in $w_{t+1}$, correlated through the Stolper-Samuelson effect to an increase of the price $p_{t+1}$.

In our analysis, the existence of a cycle of period two is associated to the critical value $\sigma^{*}$ of the elasticity of intertemporal substitution in consumption. Using equation (30), it corresponds to this flip bifurcation a critical value $\chi^{*}=$ $\left(\sigma_{2}-\sigma^{*}\right) /\left(\sigma_{2}-\sigma_{1}\right)$ of the share of the consumption good consumed by the first type of consumers. Finally, considering equation (17), the flip bifurcation can be associated to a level $\kappa^{*}=\lambda-\left(\lambda-\chi^{*}\right) /\left[(1-\delta / \rho) \epsilon_{1}\right]$ of the wealth or capital inequality between both types of consumers.

Now, we want to relate the Gini index with the occurrence of a flip bifurcation. In order to do that, we define a critical Gini index $g^{*}$ associated to the critical bifurcation value $\sigma^{*}$. Using equations (26), we obtain:

$$
g^{*}=\frac{\rho}{\rho-\delta} \frac{\rho}{\rho+\delta \varepsilon_{1}}\left(\lambda-\frac{\sigma_{2}-\sigma^{*}}{\sigma_{2}-\sigma_{1}}\right)
$$

Clearly, for the feasibility of the flip bifurcation $\kappa^{*}, \varkappa^{*}$ and $g^{*}$ are required to belong to $(0, \lambda) \cdot{ }^{5}$

\section{Heterogeneity, inequalities and fluctuations}

In this section, we begin by analyzing the role of consumers' heterogeneity on the Gini index evaluated at the steady state. Then, we will show how an increase of heterogeneity in preferences can affect the critical Gini index, i.e. the degree of inequality compatible with endogenous fluctuations.

\subsection{Heterogeneity and inequality}

On the one hand, we observe that the aggregate steady state doesn't depend on $\sigma_{1}$ and $\sigma_{2}$, because these parameters only affect the two Euler equations (13) and (14), while the aggregate variable $k$ is given by (15) and does not depend on the parameters of heterogeneity. More precisely, the aggregate production $(c, y)=(T(k, \delta k, l), \delta k)$, and derivatives $T_{1}(k, \delta k, l)$ and $T_{11}(k, \delta k, l)$ are not affected by heterogeneity. This entails also that the reduced parameters $b, \varepsilon_{1}, \varepsilon_{2}$ and $\sigma^{*}$ are invariant with respect to the heterogeneity measures.

\footnotetext{
${ }^{5}$ It is easy to show that $\kappa^{*}, \varkappa^{*}$ and $g^{*}$ are together in $(0, \lambda)$, if and only if

$$
\lambda\left[1-\varepsilon_{1}\left(1-\frac{\delta}{\rho}\right)\right]<\frac{\sigma_{2}-\sigma^{*}}{\sigma_{2}-\sigma_{1}}<\lambda
$$
}


On the other hand, at equilibrium, according to expression (26) and given the poor's share $\lambda$ in total population, the Gini index can be viewed as a function of the share $\kappa$ of capital in the poor's hands. In other words, social inequalities are equivalently captured by $g$ or $\kappa$. From (26) we derive a straightforward relation between these indices.

Corollary 3 The impact of the equilibrium capital sharing on the Gini index is negative:

$$
\frac{\partial g}{\partial \kappa}=-\frac{\rho \varepsilon_{1}}{\rho+\delta \varepsilon_{1}}<0
$$

In order to understand this simple mechanism, we need to keep in mind that capital is the only source of wealth inequality in our model. If we raise the relative part of capital owned by the poor, we lower the social inequality.

In order to stress the role of heterogeneity in preferences, let's now introduce symmetric tastes. For the sake of simplicity, to describe the role of heterogeneity on local dynamics and to capture the impact of heterogeneity on inequality through simple derivatives, we fix the midpoint

$$
\bar{\sigma} \equiv\left(\sigma_{1}+\sigma_{2}\right) / 2
$$

and we define an heterogeneity measure

$$
h \equiv\left|\sigma_{1}-\sigma_{2}\right| / 2
$$

When $\sigma_{1}<\sigma_{2}$, current and future consumption are less substitutable for the first type of agent, while they are more substitutable for the second one. The converse holds, when $\sigma_{1}>\sigma_{2}$. A simple result holds:

Corollary $4 \partial g / \partial h=0$.

Not surprisingly, heterogeneity in preferences has no influence on the steady state value of the Gini index, since, as seen above, the elasticities of intertemporal substitution in consumption $\sigma_{i}$ don't affect the steady state. In what follows, we show why, conversely, heterogeneity in preferences matters for the critical value of the Gini index through which two-period cycles occur.

\subsection{Heterogeneity and critical inequality}

This section is devoted to the role of heterogeneity in consumers' preferences on the occurrence of endogenous cycles. In the main proposition, we provide precise conditions that rely an increase of heterogeneity in preferences to a variation of the inequality level compatible with the emergence of endogenous fluctuations.

Proposition 5 Taking the midpoint $\bar{\sigma}$ as given, we raise the heterogeneity measure $h$ and we obtain the following findings.

(1) If $\sigma_{1}<\sigma_{2}$, then $\partial g^{*} / \partial h<0$ for $\bar{\sigma}<\sigma^{*}$, while $\partial g^{*} / \partial h>0$ for $\bar{\sigma}>\sigma^{*}$.

(2) If $\sigma_{1}>\sigma_{2}$, then $\partial g^{*} / \partial h>0$ for $\bar{\sigma}<\sigma^{*}$, while $\partial g^{*} / \partial h<0$ for $\bar{\sigma}>\sigma^{*}$. 
Proof. See the Appendix.

Results in Proposition 5 deserve a clear-cut interpretation of the role of heterogeneity on the critical degree of inequality, giving endogenous cycles. Two main questions arise: (i) what is the effect of the relative behavior of poor and rich $\left(\sigma_{1} \lessgtr \sigma_{2}\right)$ ?; (ii) what is the role of the average behavior $\left(\bar{\sigma} \lessgtr \sigma^{*}\right)$ ?

First, assume the rich to smooth more easily consumption over time $\left(\sigma_{1}<\right.$ $\left.\sigma_{2}\right)$. A raise in $\sigma$-heterogeneity, urges the rich to save more and, thus, to become richer, and the poor to save less. Social inequality increases and a compensating adjustment in terms of the capital sharing $\kappa$ is required, in order to restore the flip bifurcation, that is to bring back to zero $P(-1)$, the characteristic polynomial evaluated at -1 . If $\bar{\sigma}<\sigma^{*}$ (large income effects), the positive adjustment of $\kappa$ is large enough to entails a decrease in the degree of social inequality, according to Corollary 3 , which dominates the initial raise: $\partial g^{*} / \partial h<$ 0 . Conversely when $\bar{\sigma}>\sigma^{*}$ (small income effects), the increase in $\kappa$, restoring the flip, turns out to be smaller and the related decrease in the Gini index doesn't thwart the initial raise: $\partial g^{*} / \partial h>0$.

Second, when $\sigma_{1}>\sigma_{2}$, the mechanism is reversed, since, now, more heterogeneity means the poor save more and the rich less, and, in consequence, we observe an initial drop of the inequality index. If $\bar{\sigma}<\sigma^{*}$, we have a large and negative compensation in $\kappa$ and a rise in the inequality index larger than the initial fall: $\partial g^{*} / \partial h>0$. Conversely, small income effects $\left(\bar{\sigma}>\sigma^{*}\right)$ will entail a decrease of the critical inequality degree, at the very end: $\partial g^{*} / \partial h<0$.

\section{Concluding remarks}

Since the seminal work of Benahbib and Nishimura (1985), a well-known condition necessary to the appearance of endogenous cycles in a two-sector economy is a more capital intensive consumption sector. The taking into consideration of heterogeneity on the producers' side is now paralleled by the introduction of heterogeneity on the consumers' side. Our concern is understanding the interplay between these forces. More precisely, after recognizing the origin of fluctuations in productive heterogeneity, the main issue we address, is to see how the second type of diversity affects the bifurcation value through which cycles appear.

When consumers are heterogeneous there is room for thinking and studying social inequality. In our framework we are able to reformulate the bifurcation value as a critical degree of income inequality and, to keep things more understandable than in previous works, we write down the bifurcation as a Gini index.

More heterogeneity in consumers' preferences raises or lowers the critical Gini index according to the relative consumers' ability to smooth consumption over time and to the average elasticity of intertemporal substitution. The economic intuition behind is also provided.

We think that this methodology could apply also to other dynamic models, where the first welfare theorem no longer holds. A further step could be a 
study on the way heterogeneity amplifies the effects of market imperfections and changes conditions for equilibrium determinacy.

\section{Appendix}

Proof of equations (6) and (7) We maximize $F^{1}\left(k-k^{2}\left(y, l^{2}\right), l-l^{2}\right)$ with respect to $l^{2}$, taking $k, y$ and $l$ as parameters. The optimal function $l^{2}(k, y, l)$ we obtain, is replaced in $k^{2}\left(y, l^{2}\right)$ to get $k^{2}\left(y, l^{2}(k, y, l)\right)$, and the value $T(k, y, l)$ is computed, replacing these optimal factor demands $k^{2}$ and $l^{2}$ into $F^{1}\left(k-k^{2}, l-l^{2}\right)$. The envelope theorem, applied to the value, gives the slopes of the transformation frontier:

$$
\begin{aligned}
& T_{1}(k, y, l)=F_{1}^{1} \\
& T_{2}(k, y, l)=-F_{1}^{1} \partial k^{2} / \partial y \\
& T_{3}(k, y, l)=F_{2}^{1}
\end{aligned}
$$

Profit maximization and perfect factors mobility entail $F_{1}^{1}=r=p F_{1}^{2}$ and $F_{2}^{1}=$ $w=p F_{2}^{2}$. Substituting these equalities in (35)-(37) and using $\partial y / \partial k^{2}=F_{1}^{2}$, eventually we obtain equations (6)-(8).

Proof of Proposition 2 In order to characterize the local stability properties, we need to study the characteristic roots, that is the intersections of the characteristic polynomial $P(\alpha)$ with the axis of abscissas. From equations $(27)$ and (28), we know that $P(1)=0, P(0)<0$, while the form of the characteristic polynomial implies $\lim _{\alpha \rightarrow+\infty} P(\alpha)=+\infty$ and $\lim _{\alpha \rightarrow-\infty} P(\alpha)=-\infty$. Let, without loss of generality, $\alpha_{1}=1$, be the first eigenvalue. Since $\alpha_{1} \alpha_{2} \alpha_{3}=1 / \beta$, then the product of the other eigenvalues is $\alpha_{2} \alpha_{3}>1$ and at least one characteristic root, say $\alpha_{3}$, lies outside the unit circle. Since the polynomial $P$ is continuous, $P(-1)>0$ implies that $\alpha_{2}$, lies in $(-1,0)$ and $\alpha_{3}<-1$. The steady state is a saddle point. If $P(-1)<0$, then either (i) $\alpha_{2}, \alpha_{3}<-1$ or (ii) $0<\alpha_{2}<1<\alpha_{3}$ or (iii) $\alpha_{2}, \alpha_{3}>1$ or (iv) $\alpha_{2}, \alpha_{3}$ are complex and conjugated with $\left|\alpha_{2}\right|=\left|\alpha_{3}\right|>1$. Case (ii) is characterized by saddle point stability and monotonic convergence, while the other cases correspond to source configurations. Case (ii) is equivalent to a negative slope of the characteristic polynomial at 1 , that is $P^{\prime}(1)<0$. When $P(-1)=0, \alpha_{2}$ goes through -1 and the system generically undergoes a flip bifurcation.

First, assume

$$
\sigma b(1+\Delta b)-\beta E>0
$$

Inequality (38) includes the case of a more capital intensive investment sector $(b>0)$ and the one-sector case $(b=0)$. Since $b<1 / \rho$, in order to ensure prices positivity (see Benhabib and Nishimura (1985)) and always $1 / \rho<1 / \delta$, (38) implies, in general,

$$
P^{\prime}(1)=-\frac{\sigma(1-\rho b)(1-\delta b)}{\sigma b(1+\Delta b)-\beta E}<0
$$


Therefore, we obtain $0<\alpha_{2}<\alpha_{1}=1<\alpha_{3}$, that is a saddle-path stability, jointly with a monotonic convergence.

Second, suppose, to the converse:

$$
\sigma b(1+\Delta b)-\beta E<0
$$

Then $P(-1)>0$, if and only if

$$
\sigma(1+(1+\Delta) b)[1+(1 / \beta+\Delta) b]-2(1+\beta) E>0
$$

In order to locate the eigenvalues with respect to the unit circle, we require to know at first the position of the relative capital intensity difference $b$ with respect to its ranked critical points: $-\infty,-1 / \Delta,-1 /(1+\Delta),-1 /(1 / \beta+\Delta), 0,1 / \rho$, and, second, the position of the aggregate elasticity of intertemporal substitution $\sigma$ with respect to its own critical points $\sigma^{*}, \sigma^{* *}$. Crossing the intervals, we obtain an exhaustive list of cases and subcases.

(1) $-\infty<b<-1 / \Delta$. Then (38) holds, that is $0<\alpha_{2}<\alpha_{1}=1<\alpha_{3}$ (saddle-path stability and monotonic convergence).

(2) Let now $-1 / \Delta<b<-1 /(1+\Delta)$. Two subcases hold according to the values of the elasticity of intertemporal substitution.

(2.1) If $\sigma<\sigma^{* *}$, then (38) holds, that is $0<\alpha_{2}<\alpha_{1}=1<\alpha_{3}$ (saddle-path stability and monotonic convergence).

(2.2) If $\sigma^{* *}<\sigma$, then (39) and (40) are satisfied: $P(-1)>0$ and $\alpha_{3}<-1<\alpha_{2}<0<\alpha_{1}=1$ (saddle-path stability and oscillating convergence).

(3) Let $-1 /(1+\Delta)<b<-1 /(1 / \beta+\Delta)$. In this case, under $b<1 / \rho$ (prices positivity) always $\sigma^{* *}<\sigma^{*}$. Three subcases matter according to the values of the elasticity of intertemporal substitution.

(3.1) If $\sigma<\sigma^{* *}$, then (38) is satisfied. Therefore $0<\alpha_{2}<\alpha_{1}=1<\alpha_{3}$ (saddle-path stability and monotonic convergence).

(3.2) If $\sigma^{* *}<\sigma<\sigma^{*}$, then (39) and (40) are satisfied, that is $P(-1)>$ 0 and $\alpha_{3}<-1<\alpha_{2}<0<\alpha_{1}=1$ (saddle-path stability and oscillating convergence).

(3.3) If $\sigma^{* *}<\sigma^{*}<\sigma$, then (39) holds, while (40) doesn't. Therefore $P(-1)<0$ and, since $P^{\prime}(1)>0$, then, as above, either (i) $\alpha_{2}, \alpha_{3}<-1$ or (iii) $\alpha_{2}, \alpha_{3}>1$ or (iv) $\alpha_{2}, \alpha_{3}$ are complex and conjugated with $\left|\alpha_{2}\right|=\left|\alpha_{3}\right|>1$. All these cases correspond to source configurations. When $\sigma$ goes through $\sigma^{*}$, the system undergoes a flip bifurcation. By continuity with respect to the case 3.2, we have that, in a neighborhood of $\sigma^{*}$, as soon as $\sigma$ increases through $\sigma^{*}$, then $\alpha_{2}$ decreases through -1 , while $\alpha_{3}<-1$ and $\alpha_{1}=1$.

(4) Let $-1 /(1 / \beta+\Delta)<b<0$. Two subcases matter according to the values of the elasticity of intertemporal substitution.

(4.1) If $\sigma<\sigma^{* *}$, then (38) holds, that is $0<\alpha_{2}<\alpha_{1}=1<\alpha_{3}$ (saddle-path stability and monotonic convergence).

(4.2) If $\sigma^{* *}<\sigma$, then (39) and (40) are satisfied, that is $P(-1)>0$ and $\alpha_{3}<-1<\alpha_{2}<0<\alpha_{1}=1$ (saddle-path stability and oscillating convergence).

(5) Finally, set $0 \leq b<1 / \rho$. Then (38) holds as in point (1). Therefore: $0<\alpha_{2}<\alpha_{1}=1<\alpha_{3}$ : saddle-path stability and monotonic convergence. 
Proposition 2 sums up all these findings, without detailing the relative position of the eigenvalues, but discussing the crucial points of saddle-path stability and monotonic convergence.

Proof of Proposition 5 Let $\bar{\sigma}$ be fixed according to (33) and $g^{*}$ be defined by (32). If $\sigma_{1}<\sigma_{2}$, then $h=\left(\sigma_{2}-\sigma_{1}\right) / 2$ and $\left(\sigma_{2}-\sigma^{*}\right) /\left(\sigma_{2}-\sigma_{1}\right)=$ $\left(h+\bar{\sigma}-\sigma^{*}\right) /(2 h)$. We have:

$$
\frac{\partial g^{*}}{\partial h}=\frac{1}{2} \frac{\rho}{\rho-\delta} \frac{\rho}{\rho+\delta \varepsilon_{1}} \frac{\bar{\sigma}-\sigma^{*}}{h^{2}}
$$

We deduce that $\partial g^{*} / \partial h<0$, if and only if $\bar{\sigma}<\sigma^{*}$. Similarly, if $\sigma_{1}>\sigma_{2}$, $h=\left(\sigma_{1}-\sigma_{2}\right) / 2$ and $\left(\sigma_{2}-\sigma^{*}\right) /\left(\sigma_{2}-\sigma_{1}\right)=\left(h+\sigma^{*}-\bar{\sigma}\right) /(2 h)$, and therefore:

$$
\frac{\partial g^{*}}{\partial h}=\frac{1}{2} \frac{\rho}{\rho-\delta} \frac{\rho}{\rho+\delta \varepsilon_{1}} \frac{\sigma^{*}-\bar{\sigma}}{h^{2}}
$$

We conclude that $\partial g^{*} / \partial h>0$, if and only if $\bar{\sigma}<\sigma^{*}$.

\section{References}

Becker, R., Tsyganov, E., 2002. Ramsey Equilibrium in a Two-Sector Model with Heterogeneous Households. Journal of Economic Theory 105, 188-225.

Benhabib, J., Nishimura, K., 1985. Competitive Equilibrium Cycles. Journal of Economic Theory 35, 284-306.

Bosi, S., Magris, F., Venditti, A., 2005. Competitive Equilibrium Cycles with Endogenous Labor. Journal of Mathematical Economics 41, 325-349.

Drugeon, J.P., 1999. On the Production Possibility Frontier in Multi-Sectorial Economies. Working Paper EUREQua, 105.

Ghiglino, C., 2005. Wealth Inequality and Dynamic Stability. Journal of Economic Theory 124, 106-115.

Ghiglino, C., Olszak-Duquenne, M., 2001. Inequalities and Fluctuations in a Dynamic General Equilibrium Model. Economic Theory 17, 1-24.

Ghiglino, C., Venditti, A., 2005. Wealth Inequality and Macroeconomic Volatility in Two-Sector Economies. GREQAM Working Paper 2005-05, AixMarseille, France.

Herrendorf, B., Waldmann, R., Valentiny, A., 2000. Ruling Out Multiplicity and Indeterminacy: The Role of Heterogeneity. Review of Economic Studies 67, 295-307.

Kehoe, T., Levine, D., Romer, P., 1990. Determinacy of Equilibria in Dynamic Models with Finitely Many Consumers. Journal of Economic Theory 50, $1-21$.

Santos, S., 1992. Differentiability and Comparative Analysis in DiscreteTime Infinite-Horizon Optimization. Journal of Economic Theory 57, 222-229. 